\title{
BMJ Open Peripherally InSerted CEntral catheter dressing and securement in patients with cancer: the PISCES trial. Protocol for a $2 \times 2$ factorial, superiority randomised controlled trial
}

Claire M Rickard, ${ }^{1,2,3}$ Nicole M Marsh, ${ }^{1,2}$ Joan Webster, ${ }^{1,2}$ Nicole C Gavin, ${ }^{1,2}$ Raymond J Chan, ${ }^{1,2,4}$ Alexandra L McCarthy, ${ }^{1,3,5}$ Peter Mollee, ${ }^{1,3,6}$ Amanda J Ullman, ${ }^{1,2}$ Tricia Kleidon, ${ }^{1,7}$ Vineet Chopra, ${ }^{1,8}$ Li Zhang, ${ }^{1}$ Matthew R McGrail, ${ }^{1,9}$ Emily Larsen, ${ }^{1,2}$ Md Abu Choudhury, ${ }^{1,10}$ Samantha Keogh, ${ }^{1,2,4}$ Evan Alexandrou, ${ }^{1,11}$ David J McMillan, ${ }^{1,12}$ Merehau Cindy Mervin, ${ }^{1,13}$ David L Paterson, ${ }^{1,2,14}$ Marie Cooke, ${ }^{1}$ Gillian Ray-Barruel, ${ }^{1,2}$ Maria Isabel Castillo, ${ }^{1}$ Andrew Hallahan, ${ }^{1,7}$ Amanda Corley, ${ }^{1}$ E Geoffrey Playford ${ }^{1,3,6}$

To cite: Rickard CM, Marsh NM, Webster J, et al. Peripherally InSerted CEntral catheter dressing and securement in patients with cancer: the PISCES trial. Protocol for a $2 \times 2$ factorial, superiority randomised controlled trial. BMJ Open 2017;7:e015291. doi:10.1136/ bmjopen-2016-015291

- Prepublication history for this paper is available online. To view these files, please visit the journal online (http://dx.doi. org/10.1136/bmjopen-2016015291).

Received 1 December 2016 Revised 23 March 2017 Accepted 31 March 2017

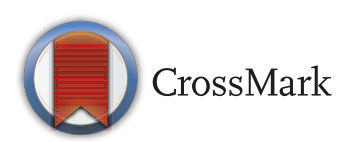

For numbered affiliations see end of article.

Correspondence to professor Claire M Rickard; c. rickard@griffith.edu.au

\section{ABSTRACT}

Introduction Around $30 \%$ of peripherally inserted central catheters (PICCs) fail from vascular, infectious or mechanical complications. Patients with cancer are at highest risk, and this increases morbidity, mortality and costs. Effective PICC dressing and securement may prevent PICC failure; however, no large randomised controlled trial (RCT) has compared alternative approaches. We designed this RCT to assess the clinical and cost-effectiveness of dressing and securements to prevent PICC failure.

Methods and analysis Pragmatic, multicentre, $2 \times 2$ factorial, superiority RCT of (1) dressings (chlorhexidine gluconate disc (CHG) vs no disc) and (2) securements (integrated securement dressing (ISD) vs securement device (SED)). A qualitative evaluation using a knowledge translation framework is included. Recruitment of 1240 patients will occur over 3 years with allocation concealment until randomisation by a centralised service. For the dressing hypothesis, we hypothesise $\mathrm{CHG}$ discs will reduce catheter-associated bloodstream infection (CABSI) compared with no CHG disc. For the securement hypothesis, we hypothesise that ISD will reduce composite PICC failure (infection (CABSI/ local infection), occlusion, dislodgement or thrombosis), compared with SED. Secondary outcomes: types of PICC failure; safety; costs; dressing/securement failure; dwell time; microbial colonisation; reversible PICC complications and consumer acceptability. Relative incidence rates of CABSI and PICC failure/100 devices and/1000 PICC days (with $95 \%$ Cls) will summarise treatment impact. KaplanMeier survival curves (and log rank Mantel-Haenszel test) will compare outcomes over time. Secondary end points will be compared between groups using parametric/nonparametric techniques; $p$ values $<0.05$ will be considered to be statistically significant.
Strengths and limitations of this study

This is the first large-scale, independent multicentre randomised controlled trial to investigate the efficacy and cost-effectiveness of peripherally inserted central catheters (PICC) dressing and securement methods in adult and paediatric cancer populations to prevent PICC complications.

- This is a pragmatic trial, with PICCs inserted and cared for by general staff in three hospitals using existing protocols, not specialist teams or researchers.

- Microbiology end points will be analysed by blinded scientists, and infection outcomes assigned by a blinded infectious disease specialist outcome assessor.

- Dressing and securement interventions cannot be blinded to clinical staff, patients or research nurses.

- Patients with existing diseased and/or non-clipped hirsute skin at the insertion point are excluded from the study so results will not be generalisable to these groups.

Ethics and dissemination Ethical approval from Queensland Health (HREC/15/QRCH/241) and Griffith University (Ref. No. 2016/063). Results will be published. Trial registration Trial registration number is: ACTRN12616000315415.

\section{INTRODUCTION}

Peripherally inserted central catheters (PICCs) are commonly placed in patients with cancer for anticancer therapies, other medicines, fluids, nutrition blood products 
and for frequent blood sampling. Although initially viewed as safer, cheaper and more durable than centrally inserted venous catheters, ${ }^{12}$ approximately $30 \%$ of PICCs fail before completion of treatment from complications, with prevalence highest in patients with cancer. ${ }^{3-8}$ As most cancer treatment is in ambulatory (outpatient) settings, PICC failure interrupting treatment poses substantial increases in lost outpatient booking times. It also depletes patients' useable veins for future treatment and can obstruct vessels long-term. Moreover, delays to chemotherapy cycles reduce treatment efficacy and can affect subsequent survival. ${ }^{9}$

PICC failure can be from infective, vascular or mechanical complications. Infections can be local insertion site infections which occur in $1 \%$ of PICCs in patients with cancer, ${ }^{10}$ or catheter-associated bloodstream infection (CABSIs) experienced by $7 \%$ hospitalised patients with cancer with PICCs, ${ }^{11}$ which increases mortality almost threefold. ${ }^{12}$ Vascular complications occur via PICC damage to the vessel endothelium, which may present as deep venous thrombosis (DVT) with severe pain and swelling, risk of thrombus detachment and potentially devastating pulmonary embolism. ${ }^{1}$ Patients with cancer frequently have increased DVT risk from coagulopathy or other haematological imbalances, and a recent meta-analysis reported DVT in 7\% of those with PICCs. ${ }^{13}$

Mechanical complications include partial or total dislodgement from the vein, occurring in 5\%-13\% of PICCs, ${ }^{3568}$ via 'drag' from multiple infusion tubes, or 'catching' on environmental structures (eg, clothing, chairs). ${ }^{415}$ Partial dislodgement malpositions the catheter tip which can cause infiltration of irritant infusion fluids and medication into tissue causing injury and potentially necrosis. ${ }^{16}$ Occlusion can result from build-up of blood or infusion products inside the PICC, or PICC compression by an irritated, swollen vein wall. Clinicians may fracture the PICC through excessive force when attempting to clear occlusion. Occlusion, with or without fracture, occurs in $4 \%-23 \%$ of PICCs. ${ }^{3568}$

It is projected that 7.2 million PICCs will be sold in the USA alone in 2017 , with revenue of US $\$ 1.26$ billion. ${ }^{17}$ Significant numbers are used worldwide, and the high incidence of PICC failure imposes substantial burden on patients and the health system. CABSI alone costs between US $\$ 12000$ and US $\$ 68000$ (2011) per episode, and 21 additional hospital days. ${ }^{18-20}$ Troubleshooting, including PICC salvage, and replacement of failed PICCs further increases costs.

\section{Current standard care for PICC dressing and securement}

Interventions to prevent PICG complications include: (i) dressings on the insertion site to prevent microbial entry and infection and (ii) securement to the skin to prevent gross dislodgement and micromotion ('pistoning', which potentiates DVT, occlusion and infection). ${ }^{21}$ Standard polyurethane (SP) transparent dressings with acrylic adhesive have been usual care since the 1980s. ${ }^{22}{ }^{23} \mathrm{~A}$ Cochrane review (7436 patients with central catheters, including 270 PICCs) found 6\% CABSI and 9\% dislodgement associated with SP dressings. ${ }^{15}$

Traditionally, PICCs were sutured for securement but securement devices (SEDs) are now preferred to avoid staff needlestick injury and PICC site infections. ${ }^{23}$ SEDs have a large adhesive padded footplate with a devicelocking clasp to reduce movement, kinking and flow impedance. $^{24}$ The one randomised controlled trial (RCT) published on SEDs reported similar dislodgement compared with sutures (12\% vs $14 \%$ ), but significantly reduced CABSI (2\% vs $12 \%$, p<0.01). ${ }^{3}$ This single-centre trial studied 170 adult patients including only 32 patients with cancer, and the inclusion and exclusion criteria were unclear. SPU were replaced every 3 days in both groups while in hospital, standard practice at that time, but different to current $5-7$ days replacement policies. ${ }^{23}$ The Cochrane review concluded there was inadequate information on generation of randomisation, attrition and noted SED manufacturer funding; they concluded further research was required, ${ }^{25}$ but this has not occurred.

\section{Novel solutions for PICC dressing and securement}

Chlorhexidine gluconate (CHG) discs placed under SP dressings release antiseptic around the PICC entry site for up to 10 days. ${ }^{21}{ }^{26}$ Systematic reviews have demonstrated effectiveness in CABSI prevention for central venous catheters, but not PICCs. ${ }^{21}$ 26-28 In patients with cancer, a single central venous catheter (CVC) trial is available, which reported lower CABSI with CHG discs than SP alone $(6 \%$ vs $11 \%, \mathrm{p}=0.02) .{ }^{29}$ The skin of patients with cancer is often impaired from steroid, radiation and/or chemotherapy treatment, ${ }^{30}$ and CHG may further irritate or damage skin. ${ }^{31}{ }^{32}$ There are also concerns about potential antimicrobial resistance, allergic reactions and systemic absorption in neonates, which may be limiting uptake. ${ }^{26} 33$

Integrated securement dressings (ISDs) are 'next-generation' SP dressings designed to replace both SP and SED. They have a tough fabric adhesive border, a second adhesive component that holds the PICC from beneath, and can be used with or without a CHG disc. A recent cohort study in PICCs $(\mathrm{n}=421)$ compared ISDs with historical SP+suture controls, finding ISDs associated with less CABSI ( $6 \%$ vs $11 \%, \mathrm{p}=0.13)$, dislodgement $(4 \%$ vs $6 \%$, $\mathrm{p}=0.27)$ and overall PICC failure $(21 \%$ vs $25 \%, \mathrm{p}=0.54) .{ }^{34}$ Occlusion in ISD-secured PICCs occurred significantly later, at 25 vs 8 days $(\mathrm{p}<0.01) .{ }^{34}$ Despite these promising findings, ISDs have not yet been tested in an RCT.

Cost-effectiveness analysis is lacking from the literature for PICC dressing and securement products, and data are needed to resolve this uncertainty. With millions of PICCs used globally, ${ }^{17}$ even small differences in cost-effectiveness could significantly reduce hospital budgets. For example, introduction of CHG discs for intensive care unit CVCs, saw net annual savings in the USA of US\$275 million to US\$2 billion for CABSI treatment. ${ }^{35-37}$

We designed a pragmatic clinical trial that has three main objectives: 
Table 1 Four groups within factorial randomised controlled trial

\begin{tabular}{lll}
\hline $\mathbf{2} \times \mathbf{2}$ factorial & Securement: SED (control) & Securement: ISD \\
\hline Dressing: no CHG disc (control) & SD+SP, no CHG disc & ISD, no CHG disc \\
Dressing: CHG disc & SD+SP with CHG disc & ISD with CHG disc \\
\hline
\end{tabular}

CHG, chlorhexidine gluconate; ISD, integrated securement dressing; SD, securement dressing; SED, securement device; SP, standard polyurethane.

1. To compare the effectiveness of PICC dressing with (i) a CHG disc and (ii) no CHG disc to prevent CABSI and adverse events;

2. To compare the effectiveness of securement with (i) an SED and (ii) an ISD to prevent PICC failure and adverse events;

3. To evaluate the acceptability of ISD and CHG discs to patients and healthcare professionals, and to identify barriers, enablers and strategies for translation of results into policy and practice.

\section{METHODS AND ANALYSIS}

This pragmatic, multicentre, $2 \times 2$ factorial, superiority RCT will test the clinical efficacy and cost-effectiveness of (1) dressings (CHG disc vs no disc) and (2) securements (SD vs ISD) (table 1). Embedded in the RCT is a formative, qualitative evaluation ${ }^{38}$ of trial products and processes using a knowledge translation framework. ${ }^{39}$

\section{Hypotheses}

Dressing hypothesis

1. The use of a CHG disc will reduce the incidence of PICC CABSI compared with the use of no disc.

\section{Securement hypothesis}

1. The use of an ISD will reduce the incidence of composite PICG failure, compared with the use of SED.

\section{Sample size and study power}

Dressing hypothesis

Our baseline CABSI is $8 \%$ with no CHG disc and we predict this incidence in the combined no $\mathrm{CHG}$ disc group. We expect $4 \%$ CABSI in the combined CHG disc groups, based on a risk ratio (RR) of 0.52 previously associated with $\mathrm{CHG}$ discs $(\mathrm{n}=7436) .{ }^{21}$ A one-sided inequality test of two proportions calculated that 602 PICCs per group would detect reduced CABSI incidence from $8 \%$ to $4 \%$ with $90 \%$ power $(\mathrm{p}=0.05$, Power Analysis and Sample Size software [PASS]) (602 CHG disc; 602 no CHG disc).

\section{To test the securement hypothesis}

Our local baseline PICC failure is $26 \%$ with SED and we predict this for the combined SED groups. We hypothesise $19 \%$ failure in the combined ISD groups $(\mathrm{RR}=0.73$ as the midpoint between $\mathrm{RR}=0.84$ associated with ISD vs $\mathrm{SP}+$ sutures in PICCs, ${ }^{34}$ and the pooled $\mathrm{RR}=0.63$ seen in our pilot trials).$^{40-42}$ A one-sided inequality test of two proportions calculated that 608 PICCs per group (608
ISD; 608 SED) could compare 26\% and 19\% PICC failure with $90 \%$ power $(\mathrm{p}=0.05$, PASS $)$.

Because of the factorial design, we used the comparison that required the larger sample (608 per group), plus $2 \%$ for potential attrition, thus 620 per group (total trial 1240). This was split so that each of the four study groups had 310 PICCs. We assumed no interaction effect between the interventions, but tested this in a Cox proportional hazards model, and analysed CHG disc effect (vs no disc) and the ISD effect (vs SED) separately (at the margins) using similar techniques. ${ }^{27} 43$ A Data Safety Monitoring Committee reviewed blinded data and serious adverse events at $n=400$ and $n=800$ to advise on (i) sample size adjustments or (ii) study stopping for efficacy in one or more arms (multiplicity adjustment, $\mathrm{p}<0.017$ ) or if one or more arms should be stopped due to futility. ${ }^{445}$ This advice was reviewed by CMR, NM, JW, MM and VC, who made the final decision to terminate the trial.

\section{Setting and sample}

After ethical, legal and governance approvals, adult and paediatric patients were recruited at three hospitals in Queensland, Australia (Lady Cilento Children's Hospital; Princess Alexandra Hospital and Royal Brisbane and Women's Hospital). Inclusion criteria were: haematological malignancy or solid tumour diagnosis; PICC required >24hours and patient/parent gave informed consent. Exclusion criteria were: non-central PICC tip placement at baseline (eg, tip in subclavian/ brachiocephalic/jugular vein); current BSI ( $<48$ hours); PICCs inserted through diseased or hirsute skin; allergy to any study product; PICCs did not already had a CHG $\mathrm{disc} /$ dressing in place. Once a patient entered the trial, consecutive PICCs were studied (as long as the inclusion/exclusion criteria were met), with all PICCs per patient following the same randomisation allocation. To ensure generalisability, PICCs inserted after-hours were also studied if these additional criteria were met: (i) <24hours since PICC insertion; (ii) no compromise in PICC function; (iii) predicted further use $\geq 24$ hours; (iv) the treating clinician agreed it was safe to replace the initial dressing/securement.

\section{Outcome measures and definitions \\ Dressing hypothesis \\ Primary outcome}

The primary outcome was CABSI: a laboratory confirmed BSI (LCBSI 1; LCBSI 2 or LCBSI 3) that is not secondary to an infection at another body site (eg, mucosal barrier 
injury LCBSI), with PICC in place for $>2$ calendar days on the day of the BSI (day of PICC placement being day 1), and the PICG was in place on the date of the event or the day before, when all elements of LCBSI, were first present together (see CDC National Healthcare Safety Network for full criteria), ${ }^{46}$ confirmed by a blinded infectious disease specialist using de-identified data.

\section{Securement hypothesis}

The primary outcome was PICC failure (composite of infection, occlusion, dislodgement, thrombosis) for the securement hypothesis.

- Infection

- CABSI: as above, or,

- Local infection (exit-site infection): microbiological diagnosis-purulent discharge (microorganism identified from exudate); or, clinical diagnosiserythema, induration and/or tenderness within $2 \mathrm{~cm}$ of PICC exit site and may be associated with other signs and symptoms of infection such as fever or purulent drainage; $B O T H$ with or without concomitant bloodstream infection, ${ }^{47}$ confirmed by a blinded infectious disease specialist.

- Occlusion

- Occlusion: $\geq 1$ lumens cannot be flushed, aspirated or resolved post-thrombolytic dwell, ${ }^{3}$ or

- Fracture: visible split in PICC material with leakage or radiographic evidence of extravasation or infiltration into tissue, causing removal. ${ }^{6}$

- Dislodgement

- Partial: change in PICG length from baseline measurement closest to skin site or PICC removal due to development of tip malposition (diagnosed radiographically and/or by site leakage on injection and/or infusion), or development of noncentral tip (eg, isolateral jugular or contralateral brachiocephalic, diagnosed radiographically), ${ }^{3}$ or

- Complete: PICC body completely leaves the vein. ${ }^{3}$

- Venous thrombosis

- Suspected: removed as too painful for patient to tolerate, ${ }^{3}$ or

- Confirmed: ultrasound-confirmed or venogramconfirmed thrombosed vessel (brachial, basilica, axillary or subclavian) at the PICC site in a symptomatic (arm pain, swelling, redness and/or tenderness over the PICC) patient, ${ }^{321}$ or diagnosis by CT, MRI or other imaging, or a symptomatic patient with a thrombus or fibrin sheath occluding $\geq 1$ lumen at PICC removal. ${ }^{10}$

Secondary outcomes (for both the dressing and securement hypotheses)

- Types of PICC failure: each failure type (local infection/ CABSI, occlusion, dislodgement or thrombosis), in addition to PICC-related bloodstream infection (laboratory confirmed matched organism from blood and catheter tip, or differential time to positivity). ${ }^{224647}$
- Safety end points: skin rash, skin tears, blisters, pruritus, local or systemic allergic reactions.

- Costs: direct costs to the hospital (in AUD) for the total episode of care, including costs of device and dressing replacement plus cost of treating PICC complications.

- Dressing/securement failure: replacement before 7 days for loose, missing, bloodstained, diaphoresis or secretion-soaked dressings/SEDs (dichotomous). ${ }^{48}$

- PICC and dressing/securement dwell time: hours from insertion/application until removal. ${ }^{40}$

- Device/skin site colonisation: $>15 \mathrm{cfu}$ growth from skin swab taken from PICC entry site, or PICC tip culture after removal. ${ }^{47}$

- Patient/parent and staff acceptability: 0-10 numerical rating scales. ${ }^{41}$

- Relative PICC failure in experimental groups: the $2 \times 2$ experimental groups were compared.

- Reversible PICC complications: complications (eg, occlusion, infection, fracture, internal malposition) that did not cause PICC failure, but required an intervention (eg, urokinase, alteplase, ethanol, PICC mended, warmed saline, reposition or 'pop' technique).

\section{Recruitment, randomisation, allocation concealment and blinding}

Research nurses (ReNs) screened patients, obtained consent after a full explanation of the trial and responded to any questions, randomised patients, educated clinical staff, patients and families, monitored protocol compliance and collected data. Central web-based randomisation with allocation concealment was patient level with 1:1:1:1 ratio between groups (randomly varied block sizes) and stratification by: (i) hospital, (ii) cancer type (haematological malignancy or solid tumour), (iii) inpatient/ outpatient status and (iii) previous PICC treatment ever (yes/no). Study products were in numbered prepacks and ReNs liaised with inserters. It is not possible to blind dressing and securements, since clinical staff must apply and monitor these, and ReNs must assess protocol compliance. The primary outcomes of CABSI/PICC failure are objective, easily and routinely collected by clinical staff (not investigators) in usual practice. Blinded microbiologists and infectious disease physicians assigned infection outcomes. A blinded statistician analysed data.

\section{Insertion and care of the PICC, dressing and securements}

ReNs were not involved in PICC insertion, application of study products or PICC care, but provided prestudy and intrastudy education to hospital staff, including user guides, to promote consistency. PICC inserters used a large sterile drape and gown, prepared skin with 2\% CHG in alcohol ${ }^{22}$ and select ed insertion site, PICC type and approach based on clinical judgement, then applied allocated products. Numbered prepacks of allocated products with a usage form was kept at the bedside and monitored by the ReNs, who confirmed the timing and reason for replacements and/or reinforcements with nursing staff. 
A protocol violation is 'the randomised product was never used to secure the PICC'. A protocol deviation is if incorrect dressing and securements are used for a proportion of the PICC dwell. Unless contraindicated (eg, irritation), deviations were corrected and the proportion of PICC dwelled with deviations, and reasons for these were noted. Study products were changed weekly and as needed, with sterile gauze used temporarily if sites bled excessively or for excessive diaphoresis. ${ }^{22}$ Use of gauze and tape or additional products (eg, extra strips of tape, bandage) was recorded for proportion of dwell time in use, and reasons for use. If CABSI was suspected by treating medical staff, percutaneously drawn and CVAD-drawn blood cultures were taken, in addition to PICC tip (if removed) culture and purulent discharge (if any) culture. PICC removal was decided by treating clinical staff as per usual practice, with no involvement by investigators.

\section{Data collection}

The ReNs collected data from electronic and other charts, using hand-held devices and a REDCap database (Research Electronic Data CAPture, Vanderbilt). The ReNs reinforced the protocol with patients and staff. A study manager checked allocation integrity, inter-rater reliability for skin and dressing assessments and monitored $100 \%$ source data verification for first patient per site, consent forms, primary outcomes and a random 5\% of other data for all patients. Site-specific hospital data at 3-6 monthly intervals noted any changes in local PICG policy or products.

At enrolment, ReNs collected patient demographics (eg, age, gender, weight); clinical factors (eg, diagnostic group, comorbidities, any infection, neutropaenia, coagulopathy, skin integrity); PICC factors (eg, insertion site, inserter discipline, technology-assisted insertion, number of insertion attempts, PICC type, size, gauge, emergency insertion, side (right/left), insertion department) and treatment factors (immunosuppressants, anticancer treatment, transfusion, antimicrobials, parenteral nutrition, continuous or intermittent therapy). Clinicians rated ease of study product application using an 11-point scale $(0=$ very difficult, $10=$ very easy), and noted previous number of applications of that product type. ${ }^{10212249}$

The ReNs followed-up study patients daily while in hospital, and weekly as outpatients (in clinic or telephone) for a maximum of 8 weeks (captured $90 \%$ of dwell data), or until PICC failure or removal if this occurred earlier. Patients contacted study staff at any time if they had questions or concerns. Each visit, dressing and securements in place, and any replacement or addition (with reason and timing) was noted. The PICC site was assessed for redness, discharge, pain, swelling, skin reactions to study products or diaphoresis. Primary, secondary and adverse outcomes (eg, skin reactions/injury) and changes in clinical, PICC or treatment factors were recorded.

At study completion, data were collected on: all infusates given through the PICG (including any lock solutions); patient mobility and cognitive status and patient/parent satisfaction with the study products $(0=$ completely dissatisfied, $10=$ completely satisfied $)$. The removing nurse rated the difficulty of product removal ( $0=$ very difficult, $10=$ very easy), and number of previous removals of that product type. The reason for removal was recorded, including any complications/failure, and PICC dwell time. ReNs prepared blinded microbiological and clinical data, and an infectious disease physician assessed infection end points. Hospital length of stay and mortality were recorded.

\section{Microbiological substudy}

A blinded microbiologist compared numbers and type of skin bacteria under the dressing/securement products at removal (purposive sample of 31 patients per group, $\mathrm{n}=124$ ). Patients were not able to be selected at random, as there were limited opportunities when ReNs were available at the time of the dressing replacement; however, we collected consecutive samples from 124 available patients at the Royal Brisbane and Women's Hospital. This ensures samples were collected by a small number of research nurses (two) with extensive training and audit to standardise sampling methods. Approximately $2 \mathrm{~cm}^{2}$ of skin at the insertion site was swabbed using a sterile cotton swabstick moistened with sterile saline for $10 \mathrm{~s}$ using back and forward motion and rolling motion, then placed in sterile tubes. Swabs and any available PICC tips were cultured on non-selective agar and incubated aerobically at $37^{\circ} \mathrm{C}$. Total number of colonies were counted and bacteria were identified at 24 hours, repeated at 72 hours for slow-growing species. ${ }^{50}$ Antibiotic resistance was tested genetically ( qacA/B and $s m r),{ }^{51}$ and CHG tolerance was tested by minimum inhibitory concentrations for each isolate using broth microdilution. ${ }^{52}$ Any positive clinical isolates from other body sites were noted from hospital records and compared with skin swab results. Specimens have been stored for future studies of infection prevention, if patients consent to this.

\section{Quantitative data analysis}

The lead investigator, statistician and study manager had access to the final dataset. Analyses and reporting followed the Consolidated Standards of reporting Trials (CONSORT) Statement. Intention-to-treat analysis occurred with patients, the unit of randomisation and PICCs, the unit of measurement. Outlying figures, missing and implausible data were cleaned. Patients who withdrew from the intervention requested to allow collection of the primary end point. Lost to follow-up patients had outcome data modelled for best-case and worst-case scenarios, with multiple imputations considered if data were missing at random. A per-protocol analysis assessed the effect of withdrawn or lost to follow-up patients, postrandomisation exclusions and protocol violations. Sensitivity analyses considered the effect of temporary protocol deviations.

Pairwise, sequential comparisons were made for CHG versus SP, and ISD versus SED. Baseline group 
comparisons were done by clinical parameters. Relative incidence rates of PICC failure per 100 devices and per 1000 PICC days (95\% CIs) summarised treatment impact, with group differences tested. Kaplan-Meier survival curves (and log rank M-H test) compared failure rates over time. Secondary end points were compared between groups using parametric and non-parametric techniques. The proportional hazards assumption was checked, and Cox regression tested the effect of group, patient, device and clinical variables on outcomes, and assessed for an interaction effect. Regression models allowed for stratification factors and clustering by site (gamma-shared frailty). ${ }^{45}$ Subgroup analysis tested for differences within and between hospital site including paediatric/adult; cancer diagnosis type and inpatient/outpatient status). $\mathrm{p}$ Values $<0.05$ were considered to be statistically significant.

\section{Estimating cost parameters}

We hypothesised significantly reduced costs in both treatments over controls from a direct hospital perspective for the episode of care (standardised AUD\$, 2019year). We quantified additional costs, benefits, net monetary benefit in the context of CABSI/PICC failure, re-insertions and the treatment costs of complications per group. Detailed resource use for PICC insertion/removal, plus dressing and securement application/removal was recorded for 100 procedures selected at random (25 per group). Staff wage costs for application, troubleshooting, replacement, consultation and equipment used were recorded. Direct observation of practice in response to CABSI or PICC failure informed total resource use and costs per group. Cost offsets due to reductions in adverse events were calculated on direct hospital costs, and for length of stay. Analysis with analysis of covariance compared mean costs per group.

\section{Qualitative data collection and analysis}

The following qualitative data complemented the trial results and informed translation:

1. Brief semi-structured interviews (approximately 10 per group, spread across sites) with patients/parents about their experience of study products, the trial and suggested improvements. ${ }^{53}$ The final sample was determined by data saturation. Interviews were audiotaped, transcribed and thematically analysed based on Norwood's framework and an inductive process. ${ }^{54}$

2. ReNs documented field notes related to positive or negative trial or study product feedback offered by any patient/parents or practitioners. Analysis was thematic as above.

3. We videorecorded approximately five episodes per group of study product use (including application, replacement and removal), with brief semi-structured interviews with the clinicians about the procedure. Final sample size was determined by data saturation, with data transcription and thematic analysis as above. Video data assessed procedural time and resource use (to inform health economic analysis), product usability and integrity of protocol delivery.

4. An Evidence Users'Reference Group was established in the final year including investigators, patients/parents, nursing and medical clinicians from cancer, infection control, infectious diseases, radiology, vascular access and policy makers. The Group reflected on trial results and qualitative data from 1 to 3 above, identified likely barriers and enablers of implementation and developed an evidence use plan with tools and strategies. ${ }^{556}$

\section{ETHICS AND DISSEMINATION OF RESULTS}

The trial had approval from the Children's Health Queensland Hospital and Health Service Human Research Ethics Committee (HREC) HREC/15/ QRCH/241, and Griffith University HREC Ref. No. 2016/063. Minor adverse events (eg, skin reaction to dressing) were treated as per usual practice with no cost to patients. Clinical trial insurance was held by the Griffith University. Written informed consent to participate was obtained from participants or representatives, including an additional option to store specimens and data for future research. Consent could be later withdrawn. Identifying details were kept confidential via assigned numeric study IDs. Serious adverse events were monitored and reported to the HREC as were any important protocol modifications. If important protocol amendments were made (eg, changes to eligibility criteria), CMR updated all investigators, HRECs, updated patient information and consent forms and updated the trial registry. A Data Safety Monitoring Committee reviewed blinded interim data and adverse events at $\mathrm{n}=400$ and 800 to advise on safety. Before qualitative interviews and video recordings, participants provided written informed consent. If patients/parents became distressed, they received initial support from the experienced qualitative reviewers and were referred to the relevant institutional contact. The trial and substudies were written by the investigators and published in peer-reviewed journals, consistent with the International Committee of Medical Journal Editors (ICMJE) Guidelines and authorship criteria.

\section{DISCUSSION}

PICC failure is unacceptably high in patients with cancer. CABSI has significant related morbidity and increases the risk of death almost threefold. ${ }^{12} 1357$ PICC failure in all its forms (eg, dislodgement) wastes millions of health dollars annually through increased procedures, treatment of complications and extended hospital days. All PICC failure results in negative patient-related outcomes, including increased pain and anxiety; delays in treatment and unnecessary exposure to the risks associated with repeated reinsertions. Older dressing and securement methods likely contribute to PICC failure, but there is inadequate data to resolve uncertainty about their efficacy 
or safety compared with newer alternatives. At present, practitioners and policy makers make decisions with uncertainty, due to lack of adequate evidence. This pragmatic, multicentre, factorial, superiority RCT will help to resolve uncertainty and inform international policy and practice.

\section{Author affiliations}

${ }^{1}$ Alliance for Vascular Access Teaching and Research (AVATAR), Menzies Health Institute Queensland, Griffith University, Brisbane, Australia

${ }^{2}$ Royal Brisbane and Women's Hospitals, Brisbane, Australia

${ }^{3}$ Princess Alexandra Hospital, Brisbane, Australia

${ }^{4}$ School of Nursing, Institute of Health and Biomedical Innovation, Queensland

University of Technology, Brisbane, Australia

${ }^{5}$ School of Nursing, University of Auckland, Auckland, New Zealand

${ }^{6}$ School of Medicine, University of Queensland, Brisbane, Australia

${ }^{7}$ Lady Cilento Children's Hospital, Brisbane, Australia

${ }^{8}$ Patient Safety Enhancement Program, University of Michigan Health System and VA Ann Arbor Health System, Ann Arbor, Michigan, USA

${ }^{9}$ School of Rural Health, Monash University, Churchill, Australia

${ }^{10}$ University of Queensland Centre for Clinical Research, Royal Brisbane and Women's Hospital Campus, Brisbane, Australia

${ }^{11}$ Western Sydney University and Liverpool Hospital, Sydney, Australia

${ }^{12}$ Inflammation and Healing Research Cluster, School of Health and Sport Sciences, University of the Sunshine Coast, Maroochydore, Australia

${ }^{13}$ Centre for Applied Health Economics, School of Medicine, Menzies Health Institute Queensland, Griffith University, Brisbane, Australia

${ }^{14}$ Centre for Clinical Research, University of Queensland, Brisbane, Australia

Correction notice This paper has been amended since it was published Online First. Owing to a scripting error, some of the publisher names in the references were replaced with 'BMJ Publishing Group'. This only affected the full text version, not the PDF. We have since corrected theseerrors and the correct publishers have been inserted into the references.

Acknowledgements CMR, NM, JW, NG, AU, TK, SK, MC and GRB are supported by the Australian National Health and Medical Research Council Centre of Research Excellence scheme. VC is supported by a career development award from the Agency for Healthcare Research and Quality (1-K08- HS022835- 01). RC is supported by the National Health and Medical Research Council Health Professional Research Fellowship scheme.

Contributor CMR, NM, JW, NG, AU, TK conceived the study. CMR, NM, JW, NG, RC, ALM, PM, AU, TK, VC, LZ, MM, EL, MAC, SK, EA, DM, MCM, DP, MC, GRB, IC, AH, EGP designed the protocol. CMR, JW, RC, PM, EGP, LZ, SK, AH, EA, AU, DM, DP, MM, MCM, NG, TC, VC, NM secured funding. CMR, NM, JW, NG, RC, ALM, PM, AU, TK, VC, LZ, MM, EL, MAC, SK, EA, DM, MCM, DP, MC, GRB, MIC, AH, AC and EGP prepared and approved the final version of the manuscript.

Competing interests CMR's employer has received on her behalf: unrestricted research and educational grants from: 3M, Adhezion, Angiodynamics, Bard, Baxter, BBraun, BD, Carefusion, Centurion Medical Products, Cook Medical, Entrotech, Flomedical, ICU Medical, Medical Australia, Medtronic, Smiths Medical, Teleflex; consultancies payments for educational lectures from 3M, Bard, BBraun, BD,Carefusion and Mayo, and expert reports from BD, ResQDevices and Smiths Medical. NM's employer has received on her behalf: consultancy payments for educational lectures based on her research from Hospira and $B D$, and unrestricted research grants from Adhezion and BD. JW: Nil. NG's employer has received on her behalf: unrestricted research grants from Baxter. RC's employer has received on his behalf: unrestricted research and educational grants from: StratPHARMA. ALM: Nil. PM's employer has received on his behalf: research grants from Celgene, Janssen and Hospira. PM is a member of Advisory Boards for Celgene, Janssen, Amgen and BMS. PM has received consultancy payments from Celgene and Amgen. AU's employer has received on her behalf: unrestricted research and educational grants from Adhezion, Angiodynamics, 3M, Carefusion, Centurion Medical Products and BBraun; consultancy payments for educational lectures based on her research from 3M and BD. TK's employer has received on her behalf: unrestricted research and educational grants from 3M, Angiodynamics, Centurion Medical Products and Baxter; consultancy payments for educational lectures from Angiodynamics and BD VC: Nil. LZ's employer has received on her behalf: an unrestricted research grant from BD. MM: Nil. EL: Nil. MAC: Nil. SK's employer has on her behalf received consultancy payments for educational lectures and unrestricted research grants from BD. EA's employer has received on his behalf: unrestricted research and educational grants from: 3M, BD, Carefusion, Cook Medical, Flo Medical. Consultancy payments for educational lectures based on his research and clinical expertise from 3M, BD, Carefusion, Teleflex, Cook Medical and Flo Medical. DM: Nil. MCM: Nil. DP: Nil. MC's employer has received on her behalf: unrestricted research and educational grants from BD, Baxter, $3 \mathrm{M}$ and Entrotech. GRB's employer has received on her behalf: unrestricted research grants from 3M, BBraun and BD/ Carefusion. Consultancy payments for educational lectures based on her research from Becton Dickinson and ResQDevices. MIC: Nil. AH: Nil. AC: Nil. EGP: Nil. No commercial entity had any role in the conception, design or funding of this study, or in the preparation of this manuscript.

Ethics approval Queensland Health (HREC/15/QRCH/241) and Griffith University (Ref. No. 2016/063).

Provenance and peer review Not commissioned; externally peer reviewed.

Data sharing statement No additional data are available.

Open Access This is an Open Access article distributed in accordance with the Creative Commons Attribution Non Commercial (CC BY-NC 4.0) license, which permits others to distribute, remix, adapt, build upon this work non-commercially, and license their derivative works on different terms, provided the original work is properly cited and the use is non-commercial. See: http://creativecommons.org/ licenses/by-nc/4.0/

(c) Article author(s) (or their employer(s) unless otherwise stated in the text of the article) 2017. All rights reserved. No commercial use is permitted unless otherwise expressly granted.

\section{REFERENCES}

1. Cotogni P, Pittiruti M. Focus on peripherally inserted central catheters in critically ill patients. World J Crit Care Med 2014;3:80-94.

2. Chopra V, Kuhn L, Coffey CE, et al. Hospitalist experiences, practice, opinions, and knowledge regarding peripherally inserted central catheters: a Michigan survey. J Hosp Med 2013;8:309-14.

3. Yamamoto AJ, Solomon JA, Soulen MC, et al. Sutureless securement device reduces complications of peripherally inserted central venous catheters. J Vasc Interv Radiol 2002;13:77-81.

4. Yap YS, Karapetis C, Lerose S, et al. Reducing the risk of peripherally inserted central catheter line complications in the oncology setting. Eur J Cancer Care 2006;15:342-7.

5. Abedin S, Kapoor G. Peripherally inserted central venous catheters are a good option for prolonged venous access in children with Cancer. Pediatr Blood Cancer 2008;51:251-5.

6. Matsuzaki A, Suminoe A, Koga Y, et al. Long-term use of peripherally inserted central venous catheters for Cancer chemotherapy in children. Support Care Cancer 2006;14:153-60.

7. Shen G, Gao Y, Wang Y, et al. Survey of the long-term use of peripherally inserted central venous catheters in children with Cancer: experience in a developing country. J Pediatr Hematol Oncol 2009;31:489-92.

8. Ullman AJ, Marsh N, Mihala G, et al. Complications of Central Venous access Devices: a systematic review. Pediatrics 2015;136:e1 331-e1344.

9. van Waart H, Stuiver MM, van Harten WH, et al. Effect of LowIntensity Physical activity and moderate- to High-Intensity Physical Exercise during adjuvant Chemotherapy on physical Fitness, Fatigue, and Chemotherapy Completion Rates: results of the PACES Randomized clinical trial. J Clin Oncol 2015;33:1918-27.

10. Worth LJ, Seymour JF, Slavin MA. Infective and thrombotic complications of central venous catheters in patients with hematological malignancy: prospective evaluation of nontunneled devices. Support Care Cancer 2009;17:811-8.

11. Chopra V, O'Horo JC, Rogers MA, et al. The risk of bloodstream infection associated with PICCs compared with CVCs in adults: a systematic review and meta-analysis. Infect Control Hosp Epidemiol 2013;34:908-18.

12. Ziegler MJ, Pellegrini DC, Safdar N. Attributable mortality of central line associated bloodstream infection: systematic review and metaanalysis. Infection 2015;43:29-36.

13. Chopra V, Anand S, Hickner A, et al. Risk of venous thromboembolism associated with peripherally inserted central catheters: a systematic review and meta-analysis. Lancet 2013;382:311-25.

14. Naimer SA, Temira F. Evaluation of techniques for intravenous catheter and tubing fixation. Mil Med 2004;169:79-81. 
15. Webster J, Gillies D, O'Riordan E, et al. Gauze and tape and transparent polyurethane dressings for central venous catheters. Cochrane Database Syst Rev 2011;11:CD003827.

16. Doellman D, Hadaway L, Bowe-Geddes LA, et al. Infiltration and extravasation: update on prevention and management. J Infus Nurs 2009;32:203-11.

17. idata Research, U.S. Vascular access Devices Market 2013. Burnaby, Canada.

18. Wilson MZ, Rafferty C, Deeter D, et al. Attributable costs of central line-associated bloodstream infections in a pediatric hematology/ oncology population. Am J Infect Control 2014;42:1157-60.

19. Warren DK, Quadir WW, Hollenbeak CS, et al. Attributable cost of catheter-associated bloodstream infections among intensive care patients in a nonteaching hospital. Crit Care Med 2006;34:2084-9.

20. Al-Rawajfah OM, Hewitt JB, Stetzer F, et al. Length of stay and charges associated with health care-acquired bloodstream infections. Am J Infect Control 2012;40:227-32.

21. Ullman AJ, Cooke M, Mitchell M, et al. Dressings and securement devices for central venous catheters (Review). Cochrane Database Syst Rev 2015;9.

22. O'Grady NP, Alexander M, Burns LA, et al. Guidelines for the prevention of intravascular catheter-related infections. Clin Infect Dis 2011;52:e162-e193.

23. Infusion nurses Society. Infusion Nursing Standards of Practice. $J$ Infus Nurs 2011;34:S1-S110.

24. Frey AM, Schears GJ. Why are we stuck on tape and suture? A review of catheter securement devices. J Infus Nurs 2006;29:34-8.

25. Yamamoto AJ, Solomon JA, Soulen MC, et al. Sutureless securement device reduces complications of peripherally inserted central venous catheters. J Vasc Interv Radiol 2002;13:77-81.

26. Timsit JF, Mimoz O, Mourvillier B, et al. Randomized controlled trial of chlorhexidine dressing and highly adhesive dressing for preventing catheter-related infections in critically ill adults. Am J Respir Crit Care Med 2012;186:1272-8.

27. Timsit JF, Schwebel C, Bouadma L, et al. Chlorhexidine-impregnated sponges and less frequent dressing changes for prevention of catheter-related infections in critically ill adults: a randomized controlled trial. JAMA 2009;301:1231-41.

28. Safdar N, O'Horo JC, Ghufran A, et al. Chlorhexidine-impregnated dressing for prevention of catheter-related bloodstream infection: a meta-analysis*. Crit Care Med 2014;42:1703-13.

29. Ruschulte $\mathrm{H}$, Franke M, Gastmeier $P$, et al. Prevention of central venous catheter related infections with chlorhexidine gluconate impregnated wound dressings: a randomized controlled trial. Ann Hematol 2009;88:267-72.

30. Rosen AC, Case EC, Dusza SW, et al. Impact of dermatologic adverse events on quality of life in 283 Cancer patients: a questionnaire study in a dermatology referral clinic. Am J Clin Dermatol 2013;14:327-33.

31. Wall JB, Divito SJ, Talbot SG. Chlorhexidine gluconate-impregnated central-line dressings and necrosis in complicated skin disorder patients. J Crit Care 2014;29:1130.e1.

32. Weitz NA, Lauren CT, Weiser JA, et al. Chlorhexidine gluconateimpregnated central access catheter dressings as a cause of erosive contact dermatitis: a report of 7 cases. JAMA Dermatol 2013;149:195-9.

33. Garland JS, Alex CP, Mueller CD, et al. A randomized trial comparing povidone-iodine to a chlorhexidine gluconate-impregnated dressing for prevention of central venous catheter infections in neonates. Pediatrics 2001;107:1431-6.

34. Saijo F, Mutoh M, Kurihara M, et al. Effect of the sutureless stablization device, SorbaView SHIELD, on the peripherally inserted central catheter. Japan J Surg Met Nutr 2014;48:107-13.

35. Schwebel C, Lucet JC, Vesin A, et al. Economic evaluation of chlorhexidine-impregnated sponges for preventing catheter-related infections in critically ill adults in the Dressing Study. Crit Care Med 2012;40:11-17.

36. Crawford AG, Fuhr JP, Rao B. Cost-benefit analysis of chlorhexidine gluconate dressing in the prevention of catheter-related bloodstream infections. Infect Control Hosp Epidemiol 2004;25:668-74.

37. Timsit JF, Schwebel C, Vegin A, et al, . Cost-benefit of a chlorhexidine impregnated sponges for prevention of catheterrelated infections in adult ICU patients. 23rd ESICM Annual Congress;2010;

38. Leon AC, Davis LL, Kraemer HC. The role and interpretation of pilot studies in clinical research. J Psychiatr Res 2011;45:626-9.

39. Graham ID, Logan J, Harrison MB, et al. Lost in knowledge translation: time for a map? J Contin Educ Health Prof 2006;26:13-24

40. Edwards M, Rickard CM, Rapchuk I, et al. A pilot trial of bordered polyurethane dressings, tissue adhesive and sutureless devices compared with standard polyurethane dressings for securing shortterm arterial catheters. Crit Care Resusc 2014;16:175-83.

41. Marsh N, Webster J, Flynn J, et al. Securement methods for peripheral venous catheters to prevent failure: a randomised controlled pilot trial. J Vasc Access 2015;16:237-44.

42. Reynolds H, Taraporewalla K, Tower M, et al. Novel technologies can provide effective dressing and securement for peripheral arterial catheters: a pilot RCT in the OT and the ICU. Aust Crit Care 2015.

43. McAlister FA, Straus SE, Sackett DL, et al. Analysis and reporting of factorial trials: a systematic review. JAMA 2003;289:2545-53.

44. Freidlin B, Korn EL, Gray R, et al. Multi-arm clinical trials of new agents: some design considerations. Clin Cancer Res 2008;14:4368-71.

45. Lee KJ, Thompson SG. The use of random effects models to allow for clustering in individually randomized trials. Clin Trials 2005;2:163-73.

46. Centers for disease control and prevention, device associated module: CLABSI. National Healthcare Safety Network, 2014:1-19.

47. Mermel LA, Allon M, Bouza E, et al. Clinical practice guidelines for the diagnosis and management of intravascular catheter-related infection: 2009 Update by the infectious diseases Society of America. Clin Infect Dis 2009;49:1-45.

48. Timsit JF, Bouadma L, Ruckly S, et al. Dressing disruption is a major risk factor for catheter-related infections. Crit Care Med 2012;40:1707-14.

49. Mollee $\mathrm{P}$, Jones $\mathrm{M}$, Stackelroth J, et al. CABSI incidence and risk factors in adults with Cancer: a prospective study. $J$ Hosp Infect 2011;78:26-30.

50. Maki DG, Weise CE, Sarafin HW. A semiquantitative culture method for identifying intravenous-catheter-related infection. N Engl J Med 1977;296:1305-9.

51. McDonnell G, Russell AD. Antiseptics and disinfectants: activity, action, and resistance. Clin Microbiol Rev 1999;12:147-79.

52. Suwantarat N, Carroll KC, Tekle T, et al. High prevalence of reduced CHG susceptibility in organisms causing CLABSI. Infect Control Hosp Epidemiol 2014;35:1183-6.

53. Plano Clark VL, Schumacher K, West C, et al. Practices for embedding an interpretive qualitative approach within a Randomized clinical trial. J Mix Methods Res 2013;7:219-42.

54. Norwood SL. Research essentials: foundations for evidence-based practice. USA: Prentice Hall, 2010.

55. Craig P, Dieppe P, Macintyre S, et al. Developing and evaluating complex interventions: the New Medical Research Council guidance. BMJ 2008;337:a1655.

56. Oakley A, Strange V, Bonell C, et al. Process evaluation in RCTs of complex interventions. Bmj 2006;332:413-6.

57. Chopra V, Anand S, Krein SL, et al. Bloodstream infection, venous thrombosis, and peripherally inserted central catheters: reappraising the evidence. Am J Med 2012;125:733-41. 